\title{
Diacronie
}

Studi di Storia Contemporanea

$N^{\circ} 14,2 \mid 2013$

Processo penale, politica, opinione pubblica (secoli XVIII-XX)

\section{Il processo a Herman Göring}

\section{Massimiliano De Prà}

\section{(2) OpenEdition}

Journals

\section{Edizione digitale}

URL: http://journals.openedition.org/diacronie/215

DOI: 10.4000/diacronie. 215

ISSN: 2038-0925

Editore

Association culturelle Diacronie

Notizia bibliografica digitale

Massimiliano De Prà, « II processo a Herman Göring », Diacronie [Online], № 14, 2 | 2013, documento 8, online dal 01 août 2013, consultato il 10 décembre 2020. URL : http://journals.openedition.org/ diacronie/215; DOI : https://doi.org/10.4000/diacronie.215 


\section{Diacronie}

N. 14 | 2|2013 Processo penale, politica, opinione pubblica (secoli XVIII-XX)

\section{8/}

\section{Il processo a Herman Göring}

Massiliano DE PRÀ*

Il processo di Norimberga rappresentò l'inizio della rinascita dopo la distruzione e le efferatezze del periodo bellico. Esso fu istituito con il proposito di punire chi avesse commesso tali nefandezze e di creare una giurisdizione internazionale che impedisse nuovamente il sorgere di tali atrocità.

L'articolo è strutturato in due parti: la prima tratta brevemente delle caratteristiche generali del processo. La seconda parte, quella principale, si incentra sul processo al più importante gerarca nazista, Hermann Göring.

\section{Il processo di Norimberga}

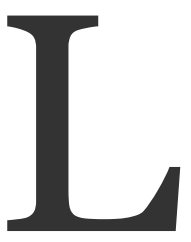

'8 maggio, dopo quasi sei anni di combattimenti, la guerra in Europa cessò con la sconfitta della Germania e la totale occupazione del suo territorio. Il conflitto aveva devastato l'Europa e la sua popolazione. Sessantacinque milioni di persone erano morte. La ferocia e il disprezzo per la vita umana caratterizzarono il periodo bellico e segnarono drasticamente la mente e l'animo di coloro che li avevano provati sulla propria pelle. Era necessario che i principali responsabili di tali atrocità fossero puniti in modo esemplare. Bisognava voltare pagina e impedire che una tale distruzione si ripetesse nuovamente in futuro.

Com'è noto, il processo di Norimberga fu un evento di portata enorme, che raccolse su di sé l'attenzione di tutto il mondo. Si svolse dal 18 ottobre 1945, l'udienza inaugurale si tenne a Berlino, fino al $1^{\circ}$ ottobre 1946 , riunendosi quasi ogni giorno. Gli imputati furono giudicati da un tribunale militare istituito appositamente per tale fine ${ }^{1}$, composto da giudici, otto in totale, e da procuratori forniti dalle quattro nazioni che

\footnotetext{
${ }^{1}$ DE FONTETTE, Francois, Il processo di Norimberga, Roma, Editori Riuniti, 1997, p. 32.
} 
avevano istituito il processo2: Stati Uniti, Gran Bretagna, Unione Sovietica e Francia. Il 13 ottobre fu eletto come presidente del tribunale il giudice inglese sir Geoffrey Lawrence.

Per la prima volta l'intero «corpo politico e sociale», che rappresentava l'ideologia di un intero movimento, e non solo singole personalità, era chiamato a giudizio per rispondere dei crimini commessi.

Sebbene anche dopo la Prima guerra mondiale si fossero svolte ampie discussioni in merito alle misure da adottare nei confronti dei crimini compiuti in guerra, fu solo dopo il secondo conflitto mondiale che nacque un diritto penale internazionale in grado di punire sistematicamente crimini di tale gravità, nonché prevenirli e scoraggiarli3 ${ }^{3}$. Il diritto assunse definitivamente la funzione di arma politica.

I crimini perpetrati durante la Seconda guerra mondiali spinsero giuristi, politici e intellettuali ad interrogarsi sulla capacità e sulla forza del diritto di impedire il perpetrarsi tali reati ${ }^{4}$ L'opinione pubblica, sconvolta dagli eventi del secondo conflitto mondiale, spalleggiava e sosteneva una tale visione del diritto. Simbolo di questo atteggiamento fu il processo di Norimberga. La corte si impegnò ad ampliare la dimensione punitiva 5 attraverso l'istituzione di nuove giurisdizioni, di cui di grandissima importanza fu quello di «crimini contro l'umanità» e «crimini contro la pace ${ }^{6}$, definizione che nacque per la prima volta nell'ambito del processo di Norimberga ${ }^{7}$.

In merito alla creazione e all'ampliamento del diritto internazionale numerose discussioni si incentrarono sul diritto come arma politica retroattiva.

I crimini nazisti furono giudicati basandosi su un corpus giuridico che, adottando i principi e le norme già emanate negli anni passati, fu creato e sviluppato a guerra finita, dopo che questi reati furono compiuti ${ }^{8}$. Infatti, l'avvocato di Göring, Stahmer, presentò, a nome dell'intera difesa, una mozione che insisteva sul fatto che fino a quel momento non era stato redatto nessun trattato internazionale che considerasse la guerra come un crimine. Quindi, secondo la difesa, in virtù del principio dell'irretroattività della legge il processo non poteva essere svolto.

\footnotetext{
2 Ibidem, p. 35 .

3 ISRAËL, Liora, Le armi del diritto, Milano, Giuffrè, 2012, p. 87.

4 SÈMELIN, Jacques, Purificare e distruggere. Usi politici dei massacri e dei genocidi, Torino, Einaudi, 2007, p. 454.

5 ZOLO, Danilo, La giustizia dei vincitori. Da Norimberga a Baghdad, Roma, Editori Laterza, 2006, p. 157.

${ }^{6}$ Ibidem, p. 27.

7 SÈMELIN, Jacques, op. cit., p. 380.

8 FLENLEY, Ralph, Storia della Germania. Dalla Riforma ai nostri giorni, Milano, Garzanti, 1969, p. 628.
} 
Questa motivazione, però, si scontrò contro un principio enunciato dalla corte, il quale asseriva che né la difesa, né l'accusa potevano contestare la legittimità del processo.

È indubbio che il processo di Norimberga si basò su un impianto accusatorio retroattivo, avendo trasgredito il principio giuridico del nulla poena sine lege, secondo il quale un'azione poteva essere perseguita solamente attraverso una legge che già esisteva al momento in cui l'azione veniva compiuta9. La legislazione esistente era inadeguata per essere utilizzata per punire crimini di una tale atrocità ed estensione, che tuttavia non potevano rimanere impuniti.

Il processo accusatorio si basò su quella che in quel momento era la concezione generale in merito alla giustizia civile e sociale. I principi su cosa fosse deprecabile o meno erano già stati ampiamente elencati dai trattati precedenti, nonché comunemente accettati dall'opinione pubblica. Quello che mancava era una legislazione internazionale. L'opera del tribunale deve essere vista come atto della creazione di questo diritto internazionale.

La retroattività fu ampiamente utilizzata dall'accusa, ma si può parlare di retroattività parziale, in quanto le leggi "create" non andavano contro ai principi e alle norme emanate in precedenza, semmai le ampliavano.

Il tribunale internazionale fu accusato, soprattutto dalla difesa tedesca, ma anche da autorevoli rappresentanti e intellettuali delle nazioni alleate, di nascondere e di dare una parvenza di legalità alla "vendetta dei vincitori nei confronti dei vinti”. La corte era composta da giudici delle quattro nazioni vincitrici. Nessuna nazione al di fuori di Stati Uniti, Gran Bretagna, Unione Sovietica e Francia era stata chiamata a prendere parte al processo, men che meno dei giudici tedeschi. Perciò la difesa gridava alla parzialità ${ }^{10} \mathrm{e}$ accusava il tribunale di aver costruito un processo su una giustizia dei vincitori stabilita ad hoc.

Il tribunale violò anche il principio dell'habeas corpus, che consisteva nell'eguaglianza di fronte alla legge. Gli imputati erano stati scelti sulla base della loro importanza all'interno della struttura gerarchica e alla posizione ricoperta nel momento in cui si era verificato un crimine internazionale ${ }^{11}$.

In merito agli stessi processi, il tribunale nascose e impedì di portare in aula materiale che avrebbe potuto coinvolgere nel processo, come accusati, esponenti e personalità sovietiche, per il trattamento nei confronti di soldati e civili tedeschi,

9 WINKLER, Heinrich, August, Grande storia della Germania. Un lungo cammino verso Occidente, vol II, Dal Terzo Reich alla repubblica di Berlino, Roma, Donzelli, 2004, p. 135.

${ }^{10}$ ZOLO, Danilo, op. cit., pp. 142-144.

${ }^{11}$ Ibidem, p. 147. 
nonché delle popolazioni dei territori occupati: non solo per salvaguardare i sovietici, ma anche per proteggere personalità americane, inglesi o francesi che avevano perpetrato alcuni dei crimini per cui erano accusati gli imputati.

L'unilateralità del processo si rese evidente anche in merito all'ostruzionismo nei confronti della difesa, che fu continuamente ostacolata nell'arco di tutto il processo, limitando agli avvocati l'accesso ai servizi di cancelleria e al materiale raccolto, di cui gli Alleati potevano liberamente usufruire.

Il processo si articolava in due distinte fasi processuali. Il principale filone riunì i principali gerarchi nazisti. A parte Hitler, Himmler, Goebbels e Mussolini, tutti deceduti, e Eichmann e Mengele, che erano riusciti a fuggire in Sudamerica, ventiquattro personalità di spicco del regime erano presenti al processo.

Per quanto concerne il processo principale, in data 30 settembre 1946 il tribunale emise dodici condanne a morte nei riguardi di Herman Göring, Joachim von Ribbentrop, Wilhelm Keitel, Ernst Kaltenbrunner, Alfred Rosenberg, Karl Hermann Frank, Wilhelm Frick, Julius Streicher, Fritz Sauckel, Alfred Jodl, Arthur Seyß-Inquart. Solo undici vennero eseguite il 16 ottobre 1946, in quanto Hermann Göring si suicidò il giorno precedente con il cianuro.

I processi secondari (secondo gruppo di processi) chiamarono a giudizio criminali di guerra minori. All'interno di questo filone vennero celebrati i processi ai medici nazisti e contro i giuristi e le istanze economiche che si erano resi colpevoli di sfruttamento della manodopera nei campi di concentramento, contro il Ministero degli Esteri, contro il Comando Supremo delle forze armate, contro i capi dei servizi di Sicurezza del Terzo Reich e contro altri esponenti del potere nazionalsocialista.

Le sentenze che vennero pronunciate furono nettamente diverse rispetto al processo principale. Quattordici esponenti furono condannati a morte, ma solo quattro sentenze furono eseguite. La maggior parte degli accusati fu assolta, mentre i restanti, seppur dichiarati colpevoli, non scontarono più di dieci anni di prigionia, o per buona condotta o per la grazia successiva.

Le accuse contro i nazisti si articolavano in quattro punti: crimini contro la pace, guerra d'aggressione, crimini contro il diritto di guerra e delitti contro l'umanità ${ }^{12}$, che furono raccolte all'interno della Carta di Norimberga.

12 IMT, Judgment of 1 October 1946, in The Trial of German Major War Criminals. Proceedings of the International Military Tribunal sitting at Nuremberg, Germany, Part 22 (22nd August, 1946 to 1st October, 1946), p. 12.

URL: < http://crimeofaggression.info/documents/6/1946_Nuremberg_Judgement.pdf > [consultato il 19 agosto 2013]. 
Tali accuse furono elaborate dal giudice della Corte Suprema degli Stati Uniti, Robert H. Jackson, e dai suoi collaboratori e, dopo lunghissimi negoziati con la controparte inglese, sovietica e francese ${ }^{13}$, furono stilati gli atti di accusa definitivi. Gli americani e gli inglesi si concentrarono sulla formulazione delle imputazioni riferite ai crimini contro la pace e alla guerra di aggressione, mentre sovietici e francesi si adoperarono nella stesura del rapporto accusatorio in merito ai crimini di guerra e ai crimini contro l'umanità. Venne stabilito che l'atto di accusa doveva riferirsi al periodo precedente l'8 maggio $1945^{14}$, giorno in cui terminò la guerra in Europa.

Ognuno dei quattro procedimenti accusatori era gestito da una commissione istituita ad hoc. La commissione 1, presieduta dagli inglesi, doveva occuparsi delle incriminazioni in merito alla guerra offensiva. I russi, che presiedevano la commissione 2, dovevano occuparsi dell'accusa di crimini di guerra e dei crimini contro l'umanità. Le atrocità commesse in Europa occidentale, nell'area che pochi anni dopo avrebbe fatto parte del blocco occidentale, erano di pertinenza della commissione 3 e dei francesi. Infine, la commissione 4, insieme agli americani, avrebbe dovuto condurre il procedimento accusatorio in merito al complotto (conspiracy) ${ }^{15}$.

Le accuse formulate dal tribunale internazionale facevano riferimento ai massacri, alla schiavitù attraverso il lavoro coatto, alla deportazione, alla persecuzione di interi popoli e di esponenti politici, alle atrocità perpetrate prima e durante la guerra in Germania e nei territori occupati, nonché alla preparazione e alla conduzione di guerre di aggressione.

Lo sterminio degli ebrei non fu trattato a parte. Non fu creato nessun punto d'accusa specifico, ma rientrò nell'accusa di crimini contro l'umanità. L'opinione pubblica internazionale non aveva ancora una percezione della specificità del crimine nazista contro il popolo ebraico. Le informazioni provenienti dai campi di concentramento di Buchenwald e di Mathausen avevano fatto in modo che fosse noto il trattamento riservato agli ebrei, ma comunque non si era ancora compresa la reale portata della soluzione finale. A ciò deve essere aggiunto che, sebbene gli americani diffusero immediatamente la voce delle atrocità compiute nei campi di concentramento, lo stesso non fu fatto da parte dei sovietici. Quando l'armata Rossa liberò i campi di concentramento, molto più diffusi nella parte di territorio occupato dall'Unione Sovietica, tra cui figura la stessa Auschwitz, niente trapelò in merito al trattamento riservato agli ebrei. In confronto alla totalità delle persone liberate dai

13 TAYLOR, Telford, Anatomia dei processi di Norimberga, Milano, Rizzoli, 1993, p. 69.

${ }_{14}$ CATARUZZA, Marina, DEAK, Istvan, Il processo di Norimberga tra storia e giustizia, Torino, UTET, 2006, p. 7.

15 TAYLOR, Telford, op. cit., p. 97. 
campi di concentramento, gli ebrei rappresentavano una minima parte, in quanto la maggior parte era già stata uccisa nelle camere a gas. La percezione comune dell'opinione pubblica si basava su dati limitati e che quindi portava ad un'erronea valutazione del trattamento riservato al popolo ebraico. Solamente quando iniziarono ad affluire dati e documenti più numerosi in merito alla politica nazista contro gli ebrei, l'opinione pubblica poté finalmente rendersi conto della portata tragica dell'evento.

Benché nessuno avesse dei dubbi che i nazisti avessero commesso atrocità sconvolgenti, la principale difficoltà del tribunale internazionale non fu il reperimento delle prove, dal momento che se ne trovarono a migliaia, ma rendere chiaro alla corte la diretta partecipazione dei singoli nazisti o gruppi di nazisti alle atrocità o, nel caso del processo ai gerarchi, che avessero partecipato alla stesura di questi piani o che ne fossero a conoscenza.

Per quanto riguarda le prime due accuse, crimini contro la pace e guerra d'aggressione, le più importanti imputazioni si riferirono alle attività per sovvertire la pace mondiale (conspiracy). Tra di esse si potevano annovere l'invasione dell'Austria, della Cecoslovacchia, della Polonia e dell'Unione Sovietica. La Germania era accusata di aver scatenato una guerra di proporzioni mondiali con le sue azioni aggressive, costringendo Gran Bretagna, Francia e, successivamente, Stati Uniti ad entrare in guerra $^{16}$, pur deprecando queste nazioni l'uso della forza, per dirimere le controversie internazionali, per salvaguardare la libertà e l'indipendenza delle nazioni e dei popoli, contravvenendo alle risoluzione del Patto Briand-Kellogg ${ }^{17}$, firmato anche dalla Germania, che sottoscriveva il rifiuto da parte dei firmatari dell'utilizzo della forza per risolvere i dissidi tra le nazioni. La Germania veniva inoltre accusata di aver portato avanti misure aggressive attraverso l'occupazione della zona smilitarizzata del Reno e in ragione del suo massiccio riarmo, di aver contravvenuto ai patti del trattato di Versailles.

Per quanto riguarda i crimini di guerra e i crimini contro l'umanità fu stilata una lunga lista, di cui elencherò le imputazioni principali. Le accuse misero in risalto l'atrocità e la totale mancanza di umanità di coloro che ordinarono ed eseguirono tali nefandezza, senza nessun rispetto per la vita umana.

I nazisti depredarono e distrussero il territorio sovietico occupato. Migliaia di villaggi e città furono rase al suolo, per non parlare della sofferenza causata agli abitanti di queste zone attraverso la violenza, gli assassini, le esecuzioni, la fame e i maltrattamenti. L'occupazione nazista causò in tali zone centinaia di migliaia di

${ }^{16}$ DE FONTETTE, Francois, op. cit., p. 52.

${ }_{17}$ Cfr. URL: < http://avalon.law.yale.edu/imt/judlawch.asp > [consultato il 19 agosto 2013]. 
vittime. Essi inoltre sfruttarono e schiavizzarono queste popolazioni per le esigenze economiche della Germania. Molti abitanti furono sradicati con la forza dalle loro zone per essere impiegate come manodopera coatta nel territorio tedesco o in altri luoghi.

Non ebbero nessun rispetto per i prigionieri di guerra. La maggior parte fu rinchiusa in campo di concentramento o fatta lavorare in condizioni proibitive. Furono frequenti i casi di maltrattamento fisico nei confronti dei prigionieri e di sistematica eliminazione di parte dei prigionieri catturati ${ }^{18}$.

Fu portato tra le prove l'ordine, redatto da Hitler nell'ottobre del 1942, di eliminare ogni soldato alleato, in uniforme o no, armato o disarmato, anche intenzionato ad arrendersi.

Furono denunciati poi gli esperimenti, operati da medici nazisti, effettuati utilizzando prigionieri di guerra come cavie.

Il processo di Norimberga mise sotto accusa non solo singole personalità, ma accusò e processò alcune organizzazioni nazionalsocialiste. La Gestapo, le SD, le SS ${ }^{19}$, le SA, il Gabinetto del Reich e lo Staff Generale e l'Alto Commando delle Forze Armate tedesche furono dichiarate organizzazioni criminali e furono smantellate.

Il processo, seppur ottenne il plauso generale per le finalità che si prefiggeva, scatenò pesanti critiche per la modalità con cui si svolse.

Una prima mancanza che venne immediatamente denunciata fu lo scarso numero di esponenti del Terzo Reich chiamati a giudizio. Il processo principale contò solo 24 imputati, mentre si raggiunsero appena i duecento se si sommano le persone interessate nei processi secondari: un numero estremamente esiguo paragonato alla vastità e brutalità dei crimini nazionalsocialisti.

Molte critiche si incentrarono sulla bontà delle formulazioni finali. Solo dodici gerarchi furono condannati a morte. Per quanto riguarda i processi secondari, in genere, gli imputati furono condannati per collaborazionismo oppure furono assolti.

Piovvero lamentele nei confronti della limitata apertura del processo di Norimberga. Non ci si curò minimamente delle esecuzioni sommarie e dei processi nei confronti di collaborazionisti che si verificarono al di fuori dei confini tedeschi, senza contare le migliaia di persone che furono incarcerate, internate nei campi di prigionia, espulse o deportate mentre erano in atto le sessioni di Norimberga.

\footnotetext{
${ }^{18}$ IMT, op. cit., p. 59.

URL: < http://crimeofaggression.info/documents/6/1946_Nuremberg_Judgement.pdf > [consultato il 19 agosto 2013].

${ }^{19}$ SCHNABEL, Reimund, Il Disonore dell'uomo. I documenti delle SS, Milano, Lerici, 1966, pp. 292-301.
} 
Il tribunale si focalizzò interamente contro esponenti della Germania nazista, evitando di prendersi la responsabilità di giudicare «esponenti non tedeschi» che, allo stesso modo della controparte nazista, meritavano di essere giudicati.

Né il tribunale di Norimberga, né quelli coevi europei, riconobbero poi l'importanza storica della Shoah, riducendo l'entità reale della tragedia, e relegandola all'interno della questione «crimini contro l'umanità».

Seppure non sia in discussione l'importanza del processo, non bisogna assolutamente sottovalutare le sue mancanze, che hanno senza dubbio offuscato il significato e l'operato di uno dei più importanti processi della storia.

\section{Il processo a Hermann Göring}

La mattina del 20 novembre ebbe ufficialmente inizio il processo di Norimberga. Tutti gli imputati erano presenti al processo, tra cui anche Hermann Göring.

Durante la lettura dell'atto di accusa egli cercò di attirare l'attenzione su di sé, parlottando continuamente con gli altri imputati e accogliendo le parole dell'accusa, talvolta con sorrisi, talvolta con smorfie. Voleva affermare la sua preminenza tra gli accusati e creare un fronte comune intorno a sé. Göring fu ossessionato durante tutto l'arco del processo dalla volontà di essere considerato, sia dagli imputati che dalle persone presenti al processo, come il più autorevole degli uomini di Hitler, sia durante il nazismo che dopo. In alcuni casi sembrò che tale proposito dominasse interamente il suo animo. Per tutto il processo cercò di ribadire costantemente il suo ruolo, pur rischiando di peggiorare la sua situazione.

Durante il terzo giorno del processo, al momento della requisitoria in merito alla genesi del nazionalismo, l'avvocato, nominando i successori di Hitler, considerò erroneamente Rudolf Hess come diretto successore del Fuhrer, al posto di Göring, sebbene fosse quest'ultimo il candidato diretto a assumere il controllo del nazionalsocialismo. Al che Göring, con una reazione spropositata, iniziò a sbracciarsi in aula cercando di attirare l'attenzione su di sé, indicando se stesso e ripetendo che era lui il numero due e non Hess $^{20}$.

L'11 dicembre il generale James Donovan presentò un filmato che avrebbe dovuto descrivere l'intero periodo del nazionalsocialismo, dall'ascesa alla guerra. Göring, come altri imputati, invece che spaventarsi per le immagini, che avrebbero potuto aggravare

${ }^{20}$ TAYLOR, Telford, op. cit., p. 199. 
la sua situazione, apprezzò il filmato, e lo riempì di gloria rivedendosi "nel buon tempo andato".

Sembrava affetto da un forte senso di sconforto per non essere riuscito a diventare l'uomo più importante del Terzo Reich, una volta che Hitler stava per cadere. Un fallimento che deve aver profondamente segnato il suo animo, spingendolo a far di tutto per ottenere il riconoscimento della sua importanza all'interno del Terzo Reich, nonché a impegnarsi al massimo per ottenere lo status di leader degli imputati al processo, anche al costo di aggravare la sua posizione.

Cercò di mantenere l'autocontrollo e di mostrarsi di fronte all'accusa sicuro di sé, sebbene in alcuni casi si lasciò andare a momenti di sconforto, di visibile depressione, ma anche di ira.

Il pomeriggio del 29 novembre fu proiettato un filmato che raccoglieva le riprese degli alleati al momento della liberazione dei campi di concentramento nella zona occupata da americani, inglesi e francesi. Le immagini erano sconvolgenti. Tale visione provocò un effetto devastante sugli imputati che, probabilmente, non le avevano mai viste. Gli accusati sprofondarono in una profonda disperazione. Chi sostenne di non aver niente a che fare con atti indegni come questi, chi ripeteva di non aver mai sentito parlare di campi di concentramento, altri piangevano e si disperavano in cella. Göring, seppur mantenne maggior controllo, fu duramente colpito da tali immagini e non riuscì a nascondere l'angoscia e la paura per la sua sorte.

Durante la testimonianza di Erwin von Lahousen, aiutante dell'ammiraglio tedesco Wilhelm Canaris, vennero letti gli ordini dello stato maggiore tedesco in merito ai prigionieri di guerra sovietici. Le direttive imponevano agli ufficiali nazisti la diretta eliminazione degli ufficiali sovietici, nonché l'assassinio immediato dei commissari politici dell'esercito. Lahousen descrisse poi le condizioni disumane in cui erano tenuti i prigionieri di guerra sovietici, che ne provocarono la morte a milioni, deliberatamente promosse dall'Oberkommando der Wehrmacht (OKW) e dallo stato maggiore nazionalsocialista. In questo frangente gli osservatori raccontarono dello scatto di rabbia che Göring ebbe, una volta riportato in cella.

In data 8 gennaio 1946 il processo si focalizzò sui singoli imputati e, fino al 4 marzo, l'accusa presiedette i lavori.

Göring fu indiziato per tutti i quattro capi di accusa sopraelencati: crimini contro la pace, guerra d'aggressione, crimini contro il diritto di guerra e delitti contro l'umanità. 
L'accusa insisteva sull'importanza dell'imputato nel Terzo Reich e sulla tremenda influenza sulle decisioni di Hitler ${ }^{21}$ fino al 1943, periodo in cui i loro rapporti iniziarono a deteriorarsi a causa delle disfatte militari sul fronte orientale. Essa riteneva che l'imputato fosse stato a conoscenza e avesse partecipato a tutte le più importanti decisioni in materia politica, economica e sociale, sin dal momento in cui entrò per la prima volta all'interno del partito nazista, nel 1922.

All'interno della sezione "crimini contro la pace", che racchiudeva assieme la prima e la seconda accusa, furono enumerate una lista lunghissima di imputazioni, che ripercorrevano l'intera carriera di Göring all'interno del nazionalsocialismo.

Lo si accusava di aver partecipato attivamente alle stesura dei piani per l'annessione dell'Austria $^{22}$ - tesi sostenuta da una sua dichiarazione in cui ammetteva di aver contribuito al 100\% all'impresa -, di aver contribuito all'incorporamento della regione dei Sudeti nel Reich, di aver approvato e sostenuto le azioni militari contro la Polonia, la Francia, la Danimarca, la Norvegia e l'Unione Sovietica, nonché l'espansione nei Balcani a danno di Grecia e Jugoslavia. Venne dipinto come uno dei maggiori sostenitori della guerra contro l'Unione Sovietica, ritenuta da lui stesso la minaccia maggiore per la Germania. Egli fu il comandante in capo della Luftwaffe durante gli attacchi alle nazioni sopraelencate e pianificò e approvò le missioni dell'aviazione tedesca durante le campagne militari.

Per quanto concerne i crimini di guerra e contro l'umanità, il procedimento accusatorio si basò su un documento in cui Göring ammise la sua complicità nello sfruttamento del lavoro coatto ${ }^{23}$, in cui sottolineò la necessità di utilizzare i lavoratori stranieri per i fini economici della Germania. Richiese più volte a Himmler di fornire maggiore manodopera servile da utilizzare nelle sue fabbriche di aereoplani. Emanò direttive affinché fossero utilizzati prigionieri di guerra polacchi, russi, francesi, inglesi e di altre nazionalità come manodopera per le fabbriche di armamenti tedesche.

L'accusa presentò un documento in cui emerse un suo ordine diretto alla dirigenza dell'SD in merito a trattamenti speciali da riservare a prigionieri di guerra russi e polacchi: questi concernevano la fucilazione immediata per gli ufficiali e i commissari, ma anche per gruppi di soldati ${ }^{24}$. In tale documento si ordinava di utilizzare parte dei prigionieri per il lavoro coatto.

${ }^{21}$ IMT, op. cit., p. 101.

URL: < http://crimeofaggression.info/documents/6/1946_Nuremberg_Judgement.pdf > [consultato il 19 agosto 2013].

${ }_{22}$ IRVING, David, Göring, Il maresciallo del Reich, Milano, Mondadori, 1989, p. 249.

23 Ibidem, p. 102.

24 DE FONTETTE, Francois, op. cit., p. 78. 
Hitler assegnò a Göring il compito di gestire i territori russi e polacchi occupati, e gli conferì l'incarico di pianificarne il saccheggio.

L'accusa presentò istanze e documenti che testimoniavano e rendevano manifesto l’impegno di Göring nella persecuzione degli ebrei e nelle decisioni in merito alla «soluzione del problema ebraico». Sebbene lo sterminio degli ebrei avrebbe dovuto essere pianificato da Himmler, furono presentate numerose prove che attestavano che l'imputato non se ne era assolutamente disinteressato o rimasto inattivo.

L'intero procedimento accusatorio era incentrato sulla dimostrazione di come egli avesse partecipato, sostenuto e, in alcuni casi, fosse stato il mandante di tutte le atrocità per cui gli imputati erano stati chiamati a giudizio.

La difesa di Göring si trovò ad affrontare un lavoro tutt'altro che semplice. Le prove presentate erano di una quantità e di una chiarezza lampante. Non c'era nessun dubbio in merito alla colpevolezza dell'imputato. In aggiunta assegnargli un avvocato non fu un compito facile. Quando la corte chiese all'interessato da chi avesse intenzione di farsi difendere, rispose, in modo sprezzante, che non era interessato a farsi rappresentare da nessuno, che gli serviva solo un'interprete, in quanto reputava che in questo processo gli avvocati non servissero a nulla.

Alla fine, però, Göring accettò come avvocato un giudice di Kiel, Otto Stahmer.

La strategia difensiva messa in pratica fu un totale disastro. I teste chiamati a testimoniare in favore dell'imputato non permisero all'avvocato di ottenere un alleggerimento della pena. Furono irrilevanti per smentire le prove presentate dall'accusa.

Gli interrogatori difensivi si svilupparono tutti allo stesso modo. Non durarono più di quindici minuti e furono caratterizzati da domande precise a cui seguivano lunghe risposte da parte degli imputati.

Stahmer chiedeva ai teste di raccontare fatti o azioni che si erano verificate durante la guerra e nel periodo precedente, e loro, secondo una schema stabilito in precedenza, raccontavano le vicende cercando di dimostrare l'estraneità di Göring. Infatti il primo teste della difesa, in data 8 marzo, fu il generale dell'aeronautica Karl Bodenschatz, che era stato ufficiale di collegamento tra l'imputato e Hitler durante la guerra. Il testimone raccontò il periodo in cui conobbe l'imputato e i successivi momenti in cui si trovò a lavorare alle sue dipendenze, $\mathrm{o}$ in stretto contatto con lui, parlando largamente a favore dell'accusato.

Analogamente, il secondo teste, Erhard Milch sostenne la tesi dell'innocenza, dichiarando come questi fosse contrario alla guerra e che non sapesse niente in merito agli omicidi e agli esperimenti dei medici nazisti. 
La linea difensiva utilizzata dall'avvocato rese manifesta la sua totale impreparazione. Le tesi dei testimoni furono ripetutamente smentite e screditate dall'abilità e dall'esperienza del giudice Jackson che, durante i controinterrogatori, li costrinse sulla difensiva. Il giudice voleva convincere la giuria dell'inaffidabilità dei testimoni e delle loro dichiarazioni: pertanto cercò di metterli in imbarazzo di fronte alla corte, insinuando che avessero partecipato anche loro ai crimini di cui erano accusati gli imputati.

Per quattro ore Milch fu tartassato e messo sulla difensiva dalle insidie lanciate dal giudice. Milch era coinvolto anch'egli in molti crimini di cui erano accusati gli imputati, perciò Jackson, prove alla mano, fece pressione sul teste, costringendolo a mentire, a far finta di essersi scordato particolari del suo rapporto con il nazismo e dipingendolo come un testimone per niente affidabile.

Molti, pressati dagli attacchi del giudice, sostennero di non ricordare quello che veniva loro chiesto, cercando di ammettere il meno possibile, come fece Paul Korner, intimo amico e collaboratore di Göring.

L'incapacità di Stahmer fornì, in alcuni casi, materiale in più alla difesa per sostenere la colpevolezza dell'imputato.

Quando interrogò Kasselring, per esempio, si incentrò sul proposito di dimostrare che le incursioni aeree ordinate da Göring avessero solo obbiettivi militari. Sebbene tali incursioni fossero state nominate all'interno del testo dell'accusa, esse non sarebbero state sicuramente utilizzate come materia comprovante le accuse a carico dell'imputato, in quanto anche gli alleati avevano utilizzato il bombardamento aereo come mezzo di guerra, quindi difficilmente i giudici dell'accusa si sarebbero basati su un elemento che avrebbe potuto ritorcersi contro di loro. Anzi, Stahmer fornì al controinterrogatorio di Fyfe l'espediente per incolpare Kesselring e Göring per aver utilizzato l'aviazione contro obbiettivi civili durante il bombardamento di Rotterdam.

Il pomeriggio del 13 marzo fu chiamato a testimoniare Göring. Stahmer e l'imputato avevano per lungo tempo discusso la loro tattica. La deposizione dell'accusato doveva essere caratterizzata da una lunga serie di domande brevi e specifiche, alle quali l'imputato avrebbe dovuto rispondere brevemente o ampiamente.

Göring riferì che aveva conosciuto il Führer dopo la fine della prima guerra mondiale quando - ma l'imputato non si ricordava se una domenica di ottobre o di novembre - si recò come spettatore a una manifestazione contro l'espatrio dei leader militari tedeschi. Qui sentì per la prima volta parlare Hitler da un palco e fu immediatamente colpito dalla forza delle sue parole e si trovò d'accordo con le accuse 
di tradimento che rivolgeva alla Repubblica di Weimar ${ }^{25}$. Pochi giorni dopo egli entrò a far parte del partito denominato NSDAP.

Illustrò gli avvenimenti che portarono all'affermazione del nazismo all'interno della Germania e i passi che portarono Hitler al potere. L’interrogatorio proseguì incentrandosi sulle accuse di crimini contro la pace, di guerra e contro l'umanità.

Göring rispose ampiamente a tutte le domande di Stahmer, soffermandosi sui particolari che egli riteneva importanti. Sostenne che le Germania fosse nel giusto quando decise di riarmarsi, anche se contravveniva ai patti siglati a Versailles. Affermò che avesse tutto il diritto di riconquistare, anche con le armi, i territori sottratti alla Germania dopo la prima guerra mondiale e, allo stesso modo, «occuparne altri indispensabili alla crescita e alla grandezza del Reich». Riconobbe di aver collaborato alla stesura di piani contro la pace (Austria, Sudeti, Danzica), di essere uno dei promotori della «guerra offensiva» al fine di annettersi ampi territori per le necessità del Reich e di aver acconsentito all'invasione e all'occupazione di altri paesi. Negò, però, di essere stato favorevole all'invasione dell'Unione Sovietica, anzi, egli mise la corte al corrente della sua contrarietà a tale decisione, aggiungendo, però, che la sua opposizione al progetto era dettata da considerazioni politico-militari e non da ragioni di diritto internazionale.

Göring ammise di aver organizzato campi di concentramento, pur sostenendo di aver dato chiare istruzioni affinché non si verificassero maltrattamenti. Si assunse la responsabilità dell'istituzione di specifici campi di concentramento per gli oppositori politici, ma dal 1934, anno in cui il loro controllo passò nelle mani di Himmler, insistette di non averne saputo più niente.

Negli interrogatori rivelò di aver contribuito alla purga nei confronti delle SA, di aver partecipato all'esecuzione senza processo di persone malviste da Hitler e di aver preso importanti misure in merito alla discriminazione razziale degli ebrei. Confessò di aver sostenuto la spoliazione finanziaria nei loro confronti e di essere stato uno dei promotori della decisione di abrogare i loro diritti civili.

Per quanto concerne la deportazione dei prigionieri di guerra ammise che furono utilizzati per sopperire alle mancanze alimentari ed economiche della Germania.

In merito all'ideologia del nazionalsocialismo, negò categoricamente di aver mai proferito e sostenuto l'idea di razza superiore, ma, quando Stahmer gli rivolse domande sulla questione ebraica, non nascose di essere antisemita e di disprezzarli ampiamente. Ammise di essere un sostenitore e un promotore della decisione di separare gli ebrei

${ }_{25}$ Cfr. URL: < http://avalon.law.yale.edu/imt/o3-13-46.asp\#Goering1 > [consultato il 19 agosto 2013]. 
dai tedeschi, ma sottolineò che era contrario all'esclusione dalla società di coloro che avevano sangue misto, cioè di coloro che avevano un antenato o un genitore ebraico. Puntualizzò di aver avuto numerose conversazioni con Hitler in merito alla questione del sangue misto, constatando che la sua opposizione alla discriminazione di questi ultimi aveva spinto Hitler a considerare tale proposta. La tensione internazionale e la guerra costrinsero il Führer ad accantonare la discussione per tempi più propizi.

L'esposizione di Göring fu, a detta degli ascoltatori, di gran classe. Egli riuscì a descrivere nei minimi dettagli tutte le fasi di sviluppo storico del nazionalismo, dalla sua genesi alla sua caduta. L'imputato dimostrò di possedere una notevole capacità retorica ${ }^{26}$, tale da riuscire a riscuotere l'ammirazione e i commenti positivi di quegli ex colleghi con cui non aveva mai avuto rapporti molto amichevoli.

A parte l'eccellente dimostrazione dialettica è difficile comprendere la strategia dell'imputato e del suo avvocato.

Göring confessò di essere colpevole per la maggior parte dei crimini di cui era accusato $^{27}$, ma senza mostrare il minimo segno di pentimento, anzi, in alcuni frangenti, il sentimento d'orgoglio la faceva da padrone.

Sicuramente, strutturare la propria difesa sull'asserzione che avesse commesso tali crimini in quanto eseguiva ordini diretti, sottolineando che, se non li avesse adempiuti, avrebbe potuto rischiare severe punizioni o addirittura la vita, avrebbe potuto garantirli maggiori possibilità di ottenere uno sconto di pena.

Dalle dichiarazioni di Göring si evince la possibilità che l'imputato, ritenendo che ormai la sua pena fosse già stata stabilità, formulasse le sue parole con lo scopo di lasciare un preciso ricordo di sé alla storia. Si presentò come una delle personalità più importanti al vertice del Terzo Reich, se non la principale dopo Hitler. Non un mero esecutore degli ordini provenienti dall'alto, ma un sostenitore e promotore della maggior parte delle direttive e scelte del nazismo.

Terminato il procedimento difensivo la parola passò al giudice Jackson che procedette al controinterrogatorio dell'imputato.

«Lei si rende conto di essere probabilmente l'unica persona al mondo in grado di illustrarci i fini veri del partito nazista e le azioni segrete dei suoi capi?» - in questo modo Jackson decise di dare avvio al controinterrogatorio.

Questa volta il giudice Jackson non riuscì a mettere in piedi un'azione efficace e devastante come aveva potuto fare con i precedenti testimoni. Parte del merito era da

${ }^{26}$ IRVING, David, op. cit., p. 619.

${ }_{27}$ Ibidem, p. 627. 
attribuire all'imputato che riuscì a eludere abilmente gli attacchi del giudice, dimostrando enorme prontezza e un'abilità di eloquenza molto rara.

L'intervento di Jackson suscitò non poche critiche: buona parte del prestigio che si era guadagnato svanì molto velocemente.

Il controinterrogatorio si concluse con l'attacco di Jackson contro le pratiche naziste di sfruttamento dei prigionieri di guerra, di bombardamenti di installazioni civili e di saccheggio di opere d'arte.

Il giorno successivo, 21 marzo, la parola passò a sir David Maxwell-Fyfe, giudice inglese e politico conservatore britannico. Quest'ultimo incentrò il proprio controinterrogatorio sulla necessità di dominare il dialogo e screditare l'imputato. Si soffermò sulla vicenda della fuga degli aviatori inglesi da Stalag Luft II. Cercò in tutti i modi di coinvolgere Göring negli assassini, accusandolo di non aver fatto nulla affinché gli aviatori fuggiti non fossero uccisi. Göring comprese il pericolo che gli stava di fronte e, perciò, fu molto vago nelle risposte, sostenendo di non ricordare o di non saper rispondere alla domanda.

Alla fine Maxwell Fyfe non riuscì a provare la complicità di Göring: emerse soltanto che l'imputato non ricevette ordini da Hitler. Egli era sì a conoscenza delle direttive del Führer, ma esse erano state recapitate a Himmler, e dunque Göring non aveva nessuna autorità per bloccare o modificare gli ordini. Risultò, oltretutto, che Göring ebbe un forte alterco con Hitler in merito alla decisione del Führer di giustiziare i prigionieri evasi.

Il controinterrogatorio di Göring terminò con l'intervento del generale sovietico Rudenko che, a differenza di Jackson e Maxwell Fyfe, non cercò di dimostrare che Göring avesse nascosto dei fatti, ma sottopose all'imputato una lunga lista di documenti, chiedendogli se avesse o meno partecipato a tali atti.

In questo caso l'accusa ottenne risultati molto utili. Fondamentale nell'intervento del sovietico fu l'accento posto sulla questione del lavoro coatto. Rudenko costrinse Göring ad ammettere di non aver avuto un ruolo secondario nello sfruttamento della manodopera ma, anzi, di aver avuto responsabilità enormi all'interno delle direttive e dei piani di sfruttamento della popolazione civile e dei prigionieri militari. Le ammissioni dell'imputato durante l'interrogatorio di Rudenko costituirono la base della condanna di Göring in merito alle accuse di crimini di guerra e di crimini contro l'umanità.

Dopo il brevissimo intervento dell'accusa francese, il 22 marzo, il processo a Hermann Göring ebbe termine. Fu condannato per tutti i quattro capi d'imputazione e 
fu condannato all'impiccagione che, però, non si svolse in quanto si tolse la vita con il cianuro.

L'opinione pubblica internazionale criticò fortemente l'accusa per come aveva sostenuto il controinterrogatorio, soffermandosi ampiamente sui limiti dell'intervento di Jackson e di Maxwell Fyfe.

Ora appare chiaro che venne attribuita eccessiva importanza al controinterrogatorio. La quantità di direttive e documenti in mano all'accusa, presentati in precedenza alla giuria e alla corte, rendevano manifesta e inconfutabile l'attribuzione di colpa e la condanna dell'imputato. Addirittura l'accusa avrebbe potuto rifiutare di sostenere il controinterrogatorio. Ma l'opinione pubblica si aspettava interventi decisivi nei confronti degli imputati e non avrebbe tollerato che l'accusa avesse tralasciato di interrogare una personalità di spicco del nazionalsocialismo.

Alcuni arrivarono addirittura a proclamare il fallimento dell'intero processo a causa dell'insuccesso del controinterrogatorio. In realtà sbagliavano, in quanto una buona parte dei gerarchi, tra cui lo stesso Göring, poteva essere condannata alla pena massima stabilita dal tribunale militare solo in base alle prove e agli elementi presentati nella prima parte del processo $^{28}$, quando gli imputati ancora non erano stati chiamati a rispondere alle domande della difesa e dell'accusa.

In complesso, il processo a Göring, contribuì a scatenare le critiche che furono rivolte al processo di Norimberga nel suo complesso. Da tutti fu visto come un evento importantissimo e di portata mondiale, ma le modalità di svolgimento e gli stessi contenuti furono ritenuti inadeguati al compito che ci si apprestava a svolgere.

${ }^{28}$ DE FONTETTE, Francois, op. cit., p. 62. 


\section{* L'autore}

Massimiliano De Prà ha conseguito la Laurea Triennale in "Studi storici e filologico-letterari" presso l'Università di Trento; è attualmente iscritto alla Laurea Magistrale per frequentare in Scienze storiche dell’Università di Bologna.

URL: < http://www.studistorici.com/progett/autori/\#De Prà >

\section{Per citare questo articolo:}

DE PRÀ, Massimiliano, «ll processo a Herman Göring», Diacronie. Studi di Storia Contemporanea : Processo penale, politica, opinione pubblica (secoli XVIII-XX), 29/08/2013,

URL:<http://www.studistorici.com/2013/08/29/depra_numero_14/ >

Diacronie Studi di Storia Contemporanea $\beta$ www.diacronie.it

Risorsa digitale indipendente a carattere storiografico. Uscita trimestrale. redazione.diacronie@hotmail.it

Comitato di redazione: Marco Abram - Jacopo Bassi - Luca Bufarale - Alessandro Cattunar - Elisa Grandi - Deborah Paci - Fausto Pietrancosta - Matteo Tomasoni - Luca Zuccolo

Diritti: gli articoli di Diacronie. Studi di Storia Contemporanea sono pubblicati sotto licenza Creative Commons 2.5

Possono essere riprodotti a patto di non modificarne i contenuti e di non usarli per fini commerciali. La citazione di estratti è comunque sempre autorizzata, nei limiti previsti dalla legge. 